\section{Efeito do treinamento contrarresistência e isoflavona na densidade mineral óssea em mulheres na pós-menopausa}

\section{Effects of isoflavone and counter-resistance training on bone mineral density in postmenopausal women}

\author{
Fábio Lera Orsatti ${ }^{1}$ \\ Eliana Aguiar Petri Nahas ${ }^{2}$ \\ Jorge Nahas-Neto ${ }^{2}$ \\ Cláudio Lera Orsatti ${ }^{2}$ \\ Altamir Santos Teixeira ${ }^{3}$
}

Resumo - O estudo teve como objetivo investigar o efeito isolado e combinado do treinamento contrarresistência (TCR) e da suplementação de isoflavona da soja (ISO) sobre a densidade mineral óssea (DMO) e a remodelação óssea em mulheres na pós-menopausa (MPM). Tratou-se de estudo clínico, prospectivo, placebo-controlado, duplo-cego (ISO) e randomizado, envolvendo 80 MPM sedentárias, com idade entre 45-70 anos, randomizadas em quatro grupos (71 completaram nove meses): TCR+ISO ( $\mathrm{n}=15)$; sem TCR+ISO $(n=20)$; TCR + placebo $(n=18)$; sem TCR + placebo $(n=18)$. As participantes randomizadas no grupo ISO receberam $100 \mathrm{mg} / \mathrm{dia} / \mathrm{VO}$ (via oral) de isoflavona e as no grupo TCR realizaram sessões supervisionadas de exercícios contrarresistência (mínimo de 2 dias/ semana). Nos momentos inicial e final do estudo, a DMO do colo do fêmur e da coluna lombar foram estimadas pela absortometria radiológica de feixes duplos de energia (DXA) e a força muscular pelo teste de 1-RM. Os valores plasmáticos de telopeptídeos carboxiterminais do colágeno tipo I (CTX), osteocalcina e fator de crescimento semelhante à insulina (IGF-1) foram dosados como marcadores de remodelação óssea. Após 9 meses de intervenção não foram observados efeitos independentes ou aditivos do TCR e ISO sobre a DMO bem como sobre os valores de osteocalcina, CTX e IGF-1 ( $p>0,05)$. Houve aumento da força muscular $(+35,2 \%)$ somente nos grupos submetidos ao TCR $(p=0,02)$. Conclui-se que o TCR e ISO não apresentam efeitos combinados ou independentes sobre a DMO do fêmur e da coluna lombar e marcadores da remodelação óssea em MPM após nove meses de intervenção.

Palavras-chave: Exercício; Menopausa; Osteocalcina; Soja.

Abstract - This study aimed to investigate the independent and additive effects of counter-resistance training (RT) and soy isoflavone supplement (ISO) on bone mineral density (BMD) and bone turnover in postmenopausal women. This study used a placebo-controlled, double-blinded (soy), randomized two (ISO vs. placebo) x two (RT vs. no RT) design. Eighty sedentary postmenopausal women, aged 45-70 years, were randomly assigned to one of four groups (71 completed a 9-month intervention): $R T+I S O(n=15) ; n o R T+I S O(n=20)$; $R T+$ placebo $(n=18)$; no $R T+$ placebo $(n=18)$. Participants randomized to ISO received $100 \mathrm{mg} /$ day/oral of soy isoflavone; and those to RT attended supervised counter-resistance training sessions at least twice a week. At baseline and 9-month, BMD was estimated by dual-energy $X$-ray absorptiometry (DXA). Serum levels of C-terminal cross-linked telopeptide of type I collagen (CTX), osteocalcin, and insulin-like growth factor-1 (IGF-1) were measured as bone turnover. ANOVA with time as the repeated measure and test $t$ were used in the statistical analysis. After 9 months of intervention, neither ISO nor RT alone affected BMD at any site or levels of CTX, osteocalcin, and IGF-1 ( $p>0.05)$. ISO and RT had no additive effects on BMD and bone turnover. RT groups showed significantly increased muscle strength (+ $35.2 \%)(p=0.02)$. We found no additive effects of resistance training and soy isoflavone on bone mineral density or bone turnover in postmenopausal women after 9-months.

Key words: Exercise; Menopause; Osteocalcin; Soy.
1 Universidade Federal do Triângulo Mineiro. Instituto de Ciências da Saúde. Departamento de Ciências do Esporte. Uberaba, MG. Brasil.

2 Universidade Estadual Paulista "Júlio de Mesquita Filho". Faculdade de Medicina de Botucatu. Departamento de Ginecologia e Obstetrícia. Botucatu, SP. Brasil.

3 Universidade Estadual Paulista "Júlio de Mesquita Filho". Faculdade de Medicina. Departamento de Doenças Tropicais e Diagnóstico por Imagem. Botucatu, SP, Brasil.

Recebido em 01/10/12 Revisado em 03/01/13 Aprovado em 12/04/13 


\section{INTRODUÇÃO}

Nos primeiros anos de pós-menopausa, em reposta ao hipoestrogenismo, ocorre rápida perda de massa óssea ${ }^{1}$. Os declínios da integridade estrutural e da densidade mineral óssea (DMO) resultam em aumento do risco para osteoporose. Esta acomete cerca de 200 milhões de pessoas no mundo, sendo mais prevalente em mulheres caucasianas ${ }^{2}$. O principal resultado clínico da osteoporose é a fratura, que ocorre, principalmente, no colo de fêmur, vértebra e punho ${ }^{2}$ e, consequentemente, perda da funcionalidade e independência ${ }^{3}$. Dessa forma, a prevenção da osteoporose é considerada essencial para manutenção da saúde, qualidade de vida e independência da mulher na pós-menopausa ${ }^{4}$. Adicionalmente, a manutenção da força muscular durante o envelhecimento pode prevenir quedas e fraturas por fragilidade, diminuindo o fardo da osteoporose ${ }^{5}$. Em mulheres na pós-menopausa, a sarcopenia (perda da massa e função musculares) e a inatividade física correlacionam positivamente com a redução da $\mathrm{DMO}^{6}$. Vários estudos têm demonstrado que mulheres na pós-menopausa mantêm a capacidade de aumentar a DMO e a massa e força musculares em resposta ao exercício físico $^{7-9}$. Os exercícios aeróbios são importantes para a manutenção da saúde geral, no entanto, o treinamento com exercícios contrarresistência é mais aplicável para efeitos local-específicos do exercício³.

As isoflavonas são os principais fitoestrogênios presentes na soja, destacando a genisteína, daidzeína e gliciteína. Estes compostos não esteróides são estruturas similares ao estrogênio e ligam-se fracamente aos receptores estrogênicos (RE) $(<1 \% \text { de afinidade })^{10}$. As isoflavonas da soja ligam-se, preferencialmente, aos RE-b que são encontrados no sistema nervoso central, ossos, parede vascular e trato urogenital. Distinto do estrogênio, as isoflavonas têm pouca afinidade aos RE-a presentes na mama e útero ${ }^{11}$. Os fitoestrogênios parecem exercer efeitos benéficos à saúde óssea ${ }^{12}$. Nos estudos in vitro, a daidzeína e a genisteína promovem o crescimento e a diferenciação de osteoblastos, além de estimular a formação e prevenir a reabsorção óssea ${ }^{12,13}$. Nos estudo clínico observacional, as mulheres na pós-menopausa com alto consumo diário de isoflavona mostraram maiores valores de DMO na coluna e no quadril comparado aos seus controles ${ }^{14}$. No entanto, os dados publicados na literatura científica permanecem inconclusivos, alguns ensaios clínicos reportaram efeitos positivos para o uso de isoflavona da soja sobre a $\mathrm{DMO}^{15,16} \mathrm{e}$ outros não $\mathrm{o}^{17,18}$.

Nos estudos experimentais com ratas ooforectomizadas, a prática combinada do exercício com a isoflavona é mais efetiva na prevenção de perda óssea quando comparada aos efeitos isolados do exercício ${ }^{19,20}$. Por outro lado, os estudos clínicos relataram pouco ou nenhum efeito positivo da associação da soja ao exercício aeróbio (caminhada) na remodelação óssea ou $\mathrm{DMO}^{21,23}$. Neste sentido, o propósito do estudo foi determinar o efeito isolado e combinado do treinamento contrarresistência e suplementação de isoflavona da soja sobre a DMO e marcadores de remodelação óssea em mulheres na pós-menopausa, com a hipótese que a combinação 
das duas intervenções provocaria significante aumento na DMO e/ou na remodelação óssea.

\section{PROCEDIMENTOS METODOLÓGICOS}

\section{Delineamento do estudo e seleção da amostra}

Foi conduzido ensaio clinico, randomizado, duplo-cego, placebo-controlado com pré e pós-teste. Incluíram-se no estudo mulheres com idade entre 45-70 anos, boa saúde, sedentárias, com data de última menstruação há pelo menos doze meses e valores de hormônio folículo estimulante (FSH) maior que $40 \mathrm{mIU} / \mathrm{ml}$. Foram considerados critérios de não-inclusão: (1) dieta com alta ingestão de fibras ou soja; (2) uso de terapia hormonal (TH) ou de fitoestrogênios pelo menos seis meses prévios; (3) pressão arterial não controlada; (4) história de miopatia, neuropatia e doença esquelética; (5) história de câncer de mama, carcinoma endometrial, doença cardiovascular e tromboembolismo; (6) hemorragia vaginal não diagnosticada; (7) alcoolismo; (8) doença gastrointestinal crônica; (9) uso de medicamentos com efeitos conhecidos sobre o metabolismo ósseo. Foram consideradas sedentárias aquelas que informaram nenhuma atividade física, além de tarefas cotidianas da casa, nos últimos seis meses. Às mulheres selecionadas, foram esclarecidos os objetivos e procedimentos a que seriam submetidas e solicitadas assinaturas do Termo de Consentimento Livre e Esclarecido, exigência da resolução no 196 de Outubro de 1996 do Conselho Nacional de Saúde. O projeto de pesquisa foi encaminhado ao Comitê de Ética em Pesquisa da Faculdade de Medicina de Botucatu, recebendo parecer favorável (OF. 602/2004).

Após o período de seleção, as mulheres foram randomicamente distribuídas em quatro grupos: TCR + ISO, treinamento contrarresistência associado à isoflavona da soja $(\mathrm{n}=20)$; sem TCR + ISO, sem treinamento contrarresistência apenas isoflavona da soja $(n=20)$; TCR + placebo (PL), treinamento contrarresistência associado ao placebo $(n=20)$ e sem TCR + PL, sem treinamento contrarresistência e placebo $(n=20)$. Os examinadores e as mulheres não tiveram conhecimento prévio quanto ao uso de isoflavona ou placebo (duplo-cego). A randomização foi realizada por estatístico que desconhecia o protocolo do estudo, por meio do software GraphPad StatMate (versão 1.01, San Diego, USA). Além disso, as caixas contendo as cápsulas foram rotuladas com códigos, identificadas somente pelo estatístico.

Assim, 40 mulheres receberam $250 \mathrm{mg}$ de extrato de soja padronizado (Glicine Max ${ }^{\oplus}$ ), correspondendo a $100 \mathrm{mg} /$ dia de isoflavona, divididas em duas cápsulas de $125 \mathrm{mg}$ de extrato da soja com 50mg de isoflavona, na forma de glicosídeos. O extrato conteve, aproximadamente, $50 \%$ de genisteína e 35\% de daidzeína. As outras 40 participantes receberam duas cápsulas de lactose por dia (placebo). Todas as cápsulas foram idênticas na aparência. As mulheres foram instruídas a devolver qualquer medicamento não usado durante as visitas. 
O tempo de seguimento foi de nove meses e as avaliações foram realizadas no momento inicial e final. Foram excluídas da análise, nove mulheres, sendo cinco no grupo TCR + ISO, duas no grupo TCR + PL e duas no grupo sem TCR + PL, por motivos de doença, problemas familiares ou violação do estudo (frequência de treinamento inferior a dois dias por semana).

\section{Avaliação da força muscular (1RM)}

A força muscular foi avaliada pelo teste de uma repetição máxima (1-RM) em exercícios dinâmicos. O teste foi realizado após um período de três a cinco sessões para adaptação e familiarização com os exercícios e o teste, em dias alternados. O teste de 1RM foi composto por tentativas e intervalos de descanso de 3 a 5 minutos, até ser encontrada a carga máxima para apenas um movimento completo e com técnica adequada. Foram permitidas, no máximo, cinco tentativas por dia. Em média, foram realizadas três tentativas e um dia para determinar 1-RM. A força foi avaliada em todos os exercícios no momento inicial do estudo.

\section{Avaliação antropométrica}

Para mensuração da massa corporal, utilizou-se balança antropométrica tipo plataforma (Filizola ${ }^{\hat{a}}$, Brasil), com precisão de $0,1 \mathrm{~kg}$, com as mulheres descalças e com mínimo de roupa possível. A estatura foi determinada em estadiômetro portátil (Seca ${ }^{\oplus}$, Brasil) afixado em parede, com precisão de $0,1 \mathrm{~cm}$. Para o cálculo do índice de massa corpórea (IMC = massa corporal/ estatura ${ }^{2}$ ), empregou-se o critério da Organização Mundial da Saúde (OMS). Para a medida da circunferência da cintura (CC), foi utilizado o ponto médio entre a última costela e a crista ilíaca, determinado com as mulheres em posição horizontal, ao final da expiração, com fita inelástica dividida em centímetros e total de 1,5 metros.

\section{Densidade Mineral Óssea}

A DMO da coluna lombar $\left(\mathrm{L}_{1}-\mathrm{L}_{4}\right)$ e do colo do fêmur foi avaliada pela absortometria radiológica de feixes duplos de energia (DXA), no momento inicial e após nove meses, usando o densitômetro Hologic QDR-2000 (HologicÒ, Waltham, MA, USA). Para minimizar a variação interavaliador, todos os exames foram realizados pelo mesmo observador. O percentual de variação intra-avaliação foi de $1 \%$ para ambas DMO da coluna lombar e do colo do fêmur. A DMO foi determinada em gramas sobre área ou volume $\left(\mathrm{g} / \mathrm{cm}^{2}\right)$.

\section{Treinamento Contrarresistência}

O protocolo de exercício contrarresistência consistiu de três sessões por semana, em dias não consecutivos, por 9 meses, sob supervisão de profissionais treinados e capacitados. A ênfase do TCR foi para hipertrofia muscular. Antes de iniciar o protocolo de TCR, as participantes foram submetidas a período de aprendizado de quatro semanas, para adaptação dos aparelhos de musculação. Nesse período, foram realizados exercícios com cargas e séries mais leves, iniciando com uma série de 15 repetições entre 40\%-50\% 
de 1-RM. A progressão, para o objetivo determinado, foi gradativa até três séries de 8-12 repetições máximas (RM) com $60-80 \%$ de 1-RM.

O protocolo foi composto por exercícios dinâmicos para membros superiores e inferiores, com duração total de 50-60 min. Para os grandes grupamentos musculares (peito, costa e coxa) foram realizados dois exercícios e para os grupos musculares menores (bíceps e tríceps) um exercício. Todos os exercícios foram realizados com três séries de 8 a 12 RM. Os exercícios foram realizados na seguinte ordem: coxa (leg press, cadeira extensora e flexora dos joelhos), peito (supino e peck deck), costa (remada e puxada alta) e braço (tríceps no pulley e rosca direta). Todas as cargas foram aferidas individualmente para cada exercício, mediante o teste de 1-RM. Estas cargas sofreram reajustes periódicos, ao final de cada mês, de acordo com os ganhos de força individual. Deste modo, foram mantidas as intensidades relativas iniciais do trabalho. Para manter a mesma carga de treinamento, ao final de cada mês, as mulheres foram orientadas a reajustar (aumentar) a carga quando ultrapassassem $12 \mathrm{RM}$, retornando a zona de treinamento entre 8-12 RM. Os exercícios abdominais (três séries de 30 repetições) e panturrilha (três séries de 20 repetições com o peso corporal) complementaram o protocolo de intervenção. Foi estipulado entre 1-2 minutos de descanso entre as séries e os exercícios. Durante as sessões de treinamento, as participantes foram orientadas a realizar a ação excêntrica em dois segundos e a ação concêntrica em um segundo.

\section{Avaliação Laboratorial}

Amostras sanguíneas foram colhidas nos momentos basal e final da intervenção, pela manhã, após 12 horas de jejum. Por meio de punção venosa, obteve-se $12 \mathrm{ml}$ de sangue. $\mathrm{O}$ soro foi separado e armazenado a $-80^{\circ} \mathrm{C}$ até o momento das análises, realizada em único ensaio. $\mathrm{O} F \mathrm{FH}$, estradiol $\left(\mathrm{E}_{2}\right)$ e fator de crescimento semelhante à insulina-1 (IGF-1) foram dosados pelo imunoensaio Immulite System (DPC ${ }^{\circ}$, USA), seguindo as instruções do fabricante. $\mathrm{O}$ valor sérico do telopeptídeos carboxiterminais do colágeno tipo I (CTX) foi avaliado pelo Serum CrossLaps Assay (Nordic Bioscience Diagnostics $^{\mathrm{O}}$, Herlev, Denmark). A osteocalcina sérica foi mensurada por sandwich enzyme immunoassay (EIA) usando anticorpo policlonal contra resíduo no N-terminal 20 (aminoácido 1-20) e resíduo do N-terminal 7 (aminoácido 43-49; Biomedical Technologyò ${ }^{\circ}$ Stoughton, MA, USA). Ao final do estudo os valores séricos de genisteína e daidzeína foram determinados pelo reversed-phase high-performance liquid chromatographic (HPLC) e scanning ultravioleta (Shimadzu' ${ }^{\mathrm{O}}$, Japan).

\section{Análise estatística}

A distribuição e a homogeneidade das variáveis foram avaliadas pelos testes de Kolmogorov-Smirnov e Levene, respectivamente. As variáveis foram apresentadas como média \pm desvio-padrão. As diferenças iniciais entre os grupos das principais características foram testadas usando ANOVA-one way. Empregou-se ANOVA $(2 \times 2 \times 2)$ de medidas repetidas para testar a 
interação e o efeito da isoflavona e do TCR sobre a DMO, remodelação óssea e força muscular. A comparação das concentrações plasmáticas de isoflavonas foi realizada pelo teste $t$ de Student independente. A correlação de Pearson foi utilizada para determinar a relação entre variáveis no momento inicial do estudo. O nível escolhido de significância foi de 5\% ( $<<0,05)$.

\section{RESULTADOS}

Das 80 participantes selecionadas, 71 completaram nove meses de intervenção: TCR+ISO ( $n=15)$; sem TCR+ISO ( $n=20)$; TCR+PL $(n=18)$ e sem TCR+PL $(n=18)$. Como esperado, a taxa de desistência foi mais alta no grupo TCR $(\mathrm{n}=7)$ do que no sem TCR $(\mathrm{n}=2)$. Porém, não foi observada diferença na taxa de desistência entre os grupos ISO $(n=5)$ e Placebo $(n=$ 4). As variáveis iniciais foram submetidas à comparação estatística e estão representadas na Tabela 1. Verificou-se que os grupos foram homogêneos para todas as variáveis exceto para IMC, onde o grupo TCR+PL apresentou menor valor em relação aos demais.

Tabela 1. Comparações das características iniciais das participantes (média \pm DP).

\begin{tabular}{|c|c|c|c|c|c|}
\hline Variáveis & $\begin{array}{l}\text { TR + ISO } \\
(n=15)\end{array}$ & $\begin{array}{l}\text { TR+PL } \\
(n=18)\end{array}$ & $\begin{array}{c}\text { sem TR+ISO } \\
(n=20)\end{array}$ & $\begin{array}{c}\text { sem TR+PL } \\
(n=18)\end{array}$ & Valor de $\mathrm{p}^{*}$ \\
\hline Idade (anos) & $55,7 \pm 7,0$ & $56,6 \pm 8,8$ & $56,0 \pm 5,8$ & $55,3 \pm 8,0$ & 0,99 \\
\hline Tempo de menopausa (anos) & $7,7 \pm 5,3$ & $8,7 \pm 6,1$ & $6,5 \pm 4,9$ & $7,7 \pm 6,2$ & 0,64 \\
\hline $\mathrm{FSH}(\mathrm{mUl} / \mathrm{mL})$ & $74,0 \pm 27,7$ & $71,8 \pm 24,3$ & $63,0 \pm 21,1$ & $63,3 \pm 21,3$ & 0,15 \\
\hline$E_{2}(p g / m L)$ & $23,1 \pm 4,8$ & $21,7 \pm 4,3$ & $22,5 \pm 4,8$ & $23,3 \pm 5,8$ & 0,59 \\
\hline Cintura $(\mathrm{cm})$ & $95,7 \pm 10,1$ & $87,3 \pm 7,7$ & $94,1 \pm 12,3$ & $95,1 \pm 11,9$ & 0,09 \\
\hline IMC $\left(\mathrm{kg} / \mathrm{m}^{2}\right)$ & $30,3 \pm 4,7$ & $26,0 \pm 3,0$ & $30,1 \pm 4,7$ & $30,4 \pm 5,3$ & 0,03 \\
\hline Força muscular (kg) & $30,7 \pm 9,2$ & $31,3 \pm 6,8$ & $33,0 \pm 10,2$ & $32,7 \pm 7,9$ & 0,15 \\
\hline DMO coluna lombar $\left(\mathrm{g} / \mathrm{cm}^{2}\right)$ & $0,91 \pm 0,17$ & $0,94 \pm 0,15$ & $0,98 \pm 0,12$ & $0,91 \pm 0,08$ & 0,33 \\
\hline T-score coluna lombar (DP) & $-1,3 \pm 1,5$ & $-1,0 \pm 1,4$ & $-0,6 \pm 1,1$ & $-1,2 \pm 0,7$ & 0,32 \\
\hline DMO colo do fêmur $\left(\mathrm{g} / \mathrm{cm}^{2}\right)$ & $0,81 \pm 0,10$ & $0,77 \pm 0,12$ & $0,81 \pm 0,09$ & $0,79 \pm 0,10$ & 0,66 \\
\hline T-score colo do fêmur (DP) & $-0,9 \pm 0,9$ & $-1,2 \pm 1,2$ & $-0,8 \pm 0,9$ & $-1,0 \pm 0,9$ & 0,60 \\
\hline Osteocalcina (ng/mL) & $28,9 \pm 14,5$ & $31,0 \pm 16,6$ & $28,9 \pm 12,3$ & $28,9 \pm 11,8$ & 0,91 \\
\hline CTX (ng/mL) & $0,52 \pm 0,27$ & $0,54 \pm 0,32$ & $0,47 \pm 0,26$ & $0,49 \pm 0,23$ & 0,80 \\
\hline IGF-1 (ng/dL) & $115,3 \pm 27,1$ & $136,9 \pm 64,0$ & $114,2 \pm 34,4$ & $120,2 \pm 53,2$ & 0,44 \\
\hline
\end{tabular}

FSH = hormônio folículo estimulante; E2 = estradiol; IMC = índice de massa corporal; DM0 = densidade mineral óssea; CTX = telopeptídeo C-terminal do colágeno tipo I; IGF-1 = fator de crescimento semelhante a insulina-1; DP = desvio padrão; ISSO = isoflavona; PL = placebo; TR = treinamento contrarresistência. * Diferença significante entre os grupos se $\mathrm{p}<0,05$ (ANOVA one way)

Na Tabela 2, estão apresentados os resultados de nove meses de TCR e/ ou isoflavona (ISO) sobre a DMO, a remodelação óssea e a força muscular. Não foram observados efeitos isolados da isoflavona de soja e do TCR sobre a DMO e marcadores de remodelação óssea após a intervenção. Assim como não houve efeito da associação do TCR com a isoflavona sobre as variáveis analisadas. Observou-se aumento na força muscular apenas nos grupos submetidos ao TCR, quando comparados aos grupos não treinados, + 35,2\% (TCR+ISO e TCR+PL; $\mathrm{n}=33$ ) e -1,1\% (sem TCR+ISO 
e sem TCR+PL; $n=38$ ), respectivamente. Não foi observada alteração na força muscular quando avaliado somente o efeito da soja (Tabela 1). Houve correlação positiva, entretanto, fraca, entre DMO e a força muscular no momento inicial do estudo (coluna lombar, $r=0,31$ e colo do fêmur, $\mathrm{r}=0,30 ; \mathrm{p}<0,05)$.

Tabela 2. Efeito de nove meses de intervenção com exercício (TR) e/ou isoflavona (ISO) sobre a força muscular, densidade mineral óssea (DMO) e a remodelação óssea (média \pm DP).

\begin{tabular}{|c|c|c|c|c|c|}
\hline Variáveis & $\begin{array}{l}\text { TR +ISO } \\
(n=15)\end{array}$ & $\begin{array}{l}\text { TR+P } \\
(n=18)\end{array}$ & $\begin{array}{l}\text { Não TR+ISO } \\
(n=20)\end{array}$ & $\begin{array}{c}\text { Não TR+P } \\
(n=18)\end{array}$ & Valor de $\mathrm{p}^{*}$ \\
\hline DMO - coluna lombar $\left(\mathrm{g} / \mathrm{cm}^{2}\right)$ & & & & & $\begin{array}{l}\text { 0,50 (TR } \times \text { tempo) } \\
0,72 \text { (ISO } \times \text { tempo) } \\
0,28 \text { (TR } \times \text { ISO } \times \text { tempo) }\end{array}$ \\
\hline Inicial & $0,909 \pm 0,167$ & $0,937 \pm 0,149$ & $0,978 \pm 0,119$ & $0,911 \pm 0,081$ & \\
\hline Final & $0,896 \pm 0,158$ & $0,932 \pm 0,154$ & $0,986 \pm 0,137$ & $0,901 \pm 0,101$ & \\
\hline DMO - colo do fêmur $\left(\mathrm{g} / \mathrm{cm}^{2}\right)$ & & & & & $\begin{array}{l}\text { 0,09 (TR } \times \text { tempo) } \\
0,10 \text { (ISO } \times \text { tempo) } \\
\text { 0,20 (TR } \times \text { ISO } \times \text { tempo) }\end{array}$ \\
\hline Inicial & $0,813 \pm 0,096$ & $0,773 \pm 0,121$ & $0,807 \pm 0,091$ & $0,792 \pm 0,096$ & \\
\hline Final & $0,786 \pm 0,095$ & $0,766 \pm 0,120$ & $0,806 \pm 0,092$ & $0,792 \pm 0,095$ & \\
\hline Osteocalcina (ng/mL) & & & & & $\begin{array}{l}\text { 0,67 (TR } \times \text { tempo) } \\
0,54 \text { (ISO } \times \text { tempo) } \\
0,27 \text { (TR } \times \text { ISO } \times \text { tempo) }\end{array}$ \\
\hline Inicial & $28,9 \pm 14,5$ & $31,0 \pm 16,6$ & $28,9 \pm 12,3$ & $28,9 \pm 11,8$ & \\
\hline Final & $32,7 \pm 15,2$ & $31,2 \pm 16,2$ & $28,7 \pm 11,6$ & $30,2 \pm 11,0$ & \\
\hline CTX (ng/mL) & & & & & $\begin{array}{l}\text { 0,52 (TR } x \text { tempo) } \\
0,96 \text { (ISO } \times \text { tempo) } \\
0,96 \text { (TR } \times \text { ISO } \times \text { tempo) }\end{array}$ \\
\hline Inicial & $0,52 \pm 0,27$ & $0,54 \pm 0,32$ & $0,47 \pm 0,26$ & $0,49 \pm 0,23$ & \\
\hline Final & $0,52 \pm 0,23$ & $0,54 \pm 0,35$ & $0,50 \pm 0,28$ & $0,52 \pm 0,24$ & \\
\hline IGF-1 (ng/dL) & & & & & $\begin{array}{l}\text { 0,44 (TR } \times \text { tempo) } \\
0,81 \text { (ISO } \times \text { tempo) } \\
\text { 0,59 (TR } \times \text { ISO } \times \text { tempo) }\end{array}$ \\
\hline Inicial & $115,3 \pm 27,1$ & $136,9 \pm 64,0$ & $114,2 \pm 34,4$ & $120,2 \pm 53,2$ & \\
\hline Final & $109,8 \pm 38,2$ & $123,7 \pm 63,0$ & $111,1 \pm 41,0$ & $120,1 \pm 66,5$ & \\
\hline $\begin{array}{l}\text { Força muscular / } \\
\text { Extensão perna (Kg) }\end{array}$ & & & & & $\begin{array}{l}\text { 0,02 (TR } \times \text { tempo) } \\
0,83 \text { (ISO } \times \text { tempo) } \\
0,86 \text { (TR } \times \text { ISO } \times \text { tempo) }\end{array}$ \\
\hline Inicial & $30,0 \pm 9,2$ & $31,4 \pm 6,6$ & $32,5 \pm 9,9$ & $31,6 \pm 6,6$ & \\
\hline Final & $39,1 \pm 8,2$ & $40,7 \pm 7,4$ & $32,8 \pm 8,5$ & $32,1 \pm 5,4$ & \\
\hline
\end{tabular}

DM0 = densidade mineral óssea; $C T X=$ telopeptídeo $C$-terminal do colágeno tipo I; IGF-1 = fator de crescimento semelhante a insulina-1; DP = desvio padrão; ISSO = isoflavona; $\mathrm{PL}$ = placebo; TR = treinamento contrarresistência. ${ }^{*}$ Diferença significante entre os grupos se $\mathrm{p}<0,05$ (ANOVA)

A Tabela 3 mostra que as concentrações de daidzeína e genisteína diferiram significativamente entre grupos após a intervenção $(\mathrm{p}<0,001)$. Sete $(20 \%)$ de 35 mulheres do grupo ISO e quatro $(11,1 \%)$ de 36 do grupo PL reportaram reações adversas, principalmente, no trato gastrointestinal. Nenhuma intercorrência grave relacionada à intervenção foi informada. 
Tabela 3. Concentrações plasmáticas de daidzeína e genisteína após nove meses de intervenção (media \pm DP).

\begin{tabular}{llll}
\hline Variáveis & $\begin{array}{l}\text { Isoflavona } \\
(\mathrm{n}=35)\end{array}$ & $\begin{array}{l}\text { Placebo } \\
(\mathrm{n}=36)\end{array}$ & Valor de $\mathrm{p}^{*}$ \\
\hline Daidzeína $(\mu \mathrm{mol} / \mathrm{dl})$ & $220,4 \pm 53,5$ & $125,4 \pm 27,9$ & $<0,0001$ \\
\hline Genisteína $(\mu \mathrm{mol} / \mathrm{dl})$ & $144,3 \pm 50,5$ & $68,1 \pm 19,5$ & $<0,0001$ \\
\hline
\end{tabular}

* Diferença significante entre os grupos. $p<0,05$ (teste-t independente).

\section{DISCUSSÃO}

No presente estudo, o TCR de intensidade moderada e a suplementação de isoflavona da soja não apresentaram efeitos combinados ou independentes sobre a DMO e a remodelação óssea após nove meses de intervenção em mulheres na pós-menopausa. Alguns estudo observaram que proteína da soja contendo $96 \mathrm{mg} /$ dia de isoflavona, por $15 \mathrm{meses}$, ou $110 \mathrm{mg} / \mathrm{dia}$, por 1 ano, não afetaram a DMO da coluna lombar, do colo do fêmur e a remodelação óssea em mulheres na pós-menopausa ${ }^{17,18}$. Além disso, uma meta-análise relatou que o uso de isoflavona por, pelo menos, um ano, com dose média de $87 \mathrm{mg} / \mathrm{dia}$, não afetou a $\mathrm{DMO}$ e que doses superiores promoveram apenas tendência à pequeno benefício ${ }^{24}$. Por outro lado, a dose de $80 \mathrm{mg} /$ dia de isoflavona foi efetiva para preservar a DMO em mulheres osteopênicas por um período de 2 anos $^{25}$. Portanto, não está claro o benefício da suplementação da isoflavona sobre a DMO da coluna e/ou do fêmur em períodos inferiores há dois anos.

O efeito isolado da isoflavona nos marcadores da remodelação óssea tem sido investigado em ensaios clínicos. Um estudo, avaliando mulheres na pós-menopausa que receberam $43 \mathrm{~g}$ de proteína da soja com duas concentrações de isoflavona (97,5mg ou $135,5 \mathrm{mg})$, observou que ambas concentrações não afetaram os marcadores da remodelação óssea (CTX e osteocalcina $)^{26}$, corroborando nossos resultados. No entanto, a forma de apresentação, a dose e a biodisponibilidade da isoflavona podem ter impacto sobre os resultados. Equol é um metabólito intestinal específico da isoflavona dadzeína encontrada na soja. Existem diferenças interindividuais na habilidade para produzir equol, podendo acarretar diferenças nos efeitos da intervenção de isoflavona na saúde humana. Baseado nessa premissa e investigando os efeitos da isoflavona da soja sobre a massa óssea, um estudo sugeriu que o efeito preventivo da isoflavona na perda óssea em mulheres na pós-menopausa depende da capacidade individual de produzir equol ${ }^{27}$.

Embora o presente estudo não tenha encontrado efeito do TCR sobre a massa óssea, existem algumas evidências da eficiência TCR em mulheres na pós-menopausa. Uma meta-análise avaliou o efeito do TCR sobre a DMO em mulheres na pós-menopausa ${ }^{8}$. Na análise de 14 estudos clínicos controlados, encontrou-se aumento médio significante de $0,06 \mathrm{~g} / \mathrm{cm}^{2}$ na coluna lombar após TCR de alta intensidade. Por outro lado, no colo do fêmur, 11 estudos controlados reportaram melhora não significante de $0,01 \mathrm{~g} / \mathrm{cm}^{2}$. O Colégio Americano de Medicina do Esporte sugere que tentativas de intervenção com exercício, incluindo exercício contrarresistência progres- 
sivo de alta intensidade, podem proporcionar aumento na DMO da coluna e quadril em mulheres na pós-menopausa com ou sem o uso de terapia hormonal ${ }^{5}$. Porém, TCR de intensidade moderada não tem demonstrado o mesmo efeito. A possível explicação para esta diferença é que o mecanismo de remodelação depende da percepção da demanda funcional (força exercida no osso) ${ }^{28}$. No presente estudo, levando em conta o cuidado tomado quanto à aplicação de treinamentos de alta intensidade ( $>80 \%$ de $1 \mathrm{RM})$ em mulheres sedentárias, a progressão de sobrecarga foi aplicada. No entanto, somente $6 \%$ das mulheres progrediram para intensidade (cargas) de $80 \%$ de $1 \mathrm{RM}$, enquanto o restante permaneceu entre $60-70 \%$ de $1 \mathrm{RM}$ (intensidade moderada). Isto pode ser atribuído à variação na faixa etária (45-70 anos) das participantes, bem como o aspecto cultural quanto à realização da força (o medo). Portanto, a falta de intensidade pode ter contribuído para a não alteração da DMO com o TCR no presente estudo ${ }^{22,23}$.

Baseado em estudos experimentais que relataram benefício da associação entre isoflavona e exercício ${ }^{19-20}$ e outros que mostraram que o exercício contrarresistência é eficiente para aumentar a força e massa muscular em mulheres na pós-menopausa ${ }^{14}$, a hipótese do estudo era que a combinação das intervenções (isoflavona + TCR) proporcionaria efeitos positivos e precoces na DMO. No entanto, no presente estudo, não se observou efeitos aditivos do TCR de intensidade moderada e da isoflavona da soja sobre a DMO e a remodelação óssea após nove meses de intervenção. Um estudo relatou que a proteína da soja isolada ( $91,2 \mathrm{mg}$ de isoflavona) e o exercício de caminhada de intensidade moderada por nove meses não apresentaram efeitos aditivos na $\mathrm{DMO}$ e remodelação óssea da coluna lombar e colo do fêmur em 43 mulheres na pós-menopausa ${ }^{23}$. Outro estudo relatou que a intervenção combinada entre $75 \mathrm{mg} /$ dia de isoflavona e caminhada três vezes/semana durante um ano não apresentou efeito na $\mathrm{DMO}$ e remodelação óssea da coluna lombar e do colo do fêmur ${ }^{22}$. Coletivamente, os dados sugerem que a combinação do exercício aeróbio ou contrarresistência, com isoflavona ( $100 \mathrm{mg} / \mathrm{dia})$, não promove efeito aditivo sobre a DMO ou marcadores da remodelação óssea em períodos curtos ( $\leq 12$ meses).

Ao término do presente estudo, as mulheres engajadas no TCR ganharam força muscular, considerando que o grupo não treinado exibiu perda. O exercício contrarresistência é efetivo para restaurar a força muscular e melhorar a qualidade de vida de mulheres na pós-menopausa, ${ }^{99}$. Recomenda-se o exercício de força como intervenção importante para obter benefícios na função muscular, reduzir a frequência de quedas e manter a independência e a qualidade de vida de pessoas mais velhas ${ }^{30}$. Neste estudo, houve correlação positiva e significante entre os valores da massa muscular e da DMO no momento inicial. Embora o sistema esquelético e o muscular sejam estruturas independentes, ambos adaptam a cargas mecânicas por vias ainda não bem esclarecidas. Dessa forma, o TCR, por preservar a força muscular, pode ser efetivo na preservação da massa óssea ${ }^{5,6}$.

O presente estudo apresenta algumas limitações. Primeiro, o tamanho da amostra foi relativamente pequeno devido aos critérios de inclusão e 
natureza da intervenção (TCR) usada. Segundo, a duração da intervenção (nove meses) foi curta para resultados sobre a DMO. Terceiro, o regime de TCR de intensidade moderada utilizado, apesar de aumentar a força e a massa musculares, pode produzir um impacto reduzido na DMO e na remodelação óssea quando comparado a treinamento de alta intensidade. Quarto, embora as participantes fossem orientadas, a ingestão de alimentos com isoflavona não foi controlada, o que pode explicar a concentração plasmática de daidzeína e genistína encontrada no grupo placebo. Finalmente, a taxa de desistência variou de 2,5\% no grupo não treinado e $20 \%$ no grupo treinado. Estas taxas são altas, mas ainda comparáveis com observadas em outras tentativas envolvendo exercício ${ }^{7}$. De fato, a razão frequentemente informada para desistência foi falta de interesse para continuar com o protocolo de TCR.

\section{CONCLUSÃO}

Pode-se concluir que o TCR de intensidade moderada e a suplementação de isoflavona da soja (100mg/dia) não apresentam efeitos sobre a DMO e a remodelação óssea, de forma combinada ou independente, em mulheres na pós-menopausa, após nove meses de intervenção.

\section{REFERÊNCIAS BIBLIOGRÁFICAS}

1. Riggs BL, Khosla S, Melton LJ, 3rd. A unitary model for involutional osteoporosis: estrogen deficiency causes both type I and type II osteoporosis in postmenopausal women and contributes to bone loss in aging men. J Bone Miner Res 1998;13(5):763-73.

2. Cummings SR, Melton LJ. Epidemiology and outcomes of osteoporotic fractures. Lancet 2002;18;359(9319):1761-7.

3. Suominen H. Muscle training for bone strength. Aging Clin Exp Res 2006;18(2):85-93.

4. NAMS. Management of osteoporosis in postmenopausal women: 2010 position statement of The North American Menopause Society. Menopause 2010;17(1):2554; quiz 5-6.

5. Kohrt WM, Bloomfield SA, Little KD, Nelson ME, Yingling VR. American College of Sports Medicine Position Stand: physical activity and bone health. Med Sci Sports Exerc 2004;36(11):1985-96.

6. Orsatti FL, Nahas EA, Nahas-Neto J, Orsatti CL, Marocolo M, Barbosa-Neto O, et al. Low appendicular muscle mass is correlated with femoral neck bone mineral density loss in postmenopausal women. BMC Musculoskelet Disord 2011;12:225.

7. Bonaiuti D, Shea B, Iovine R, Negrini S, Robinson V, Kemper HC, et al. Exercise for preventing and treating osteoporosis in postmenopausal women. Cochrane Database Syst Rev 2002(3):CD000333.

8. Martyn-St James M, Carroll S. High-intensity resistance training and postmenopausal bone loss: a meta-analysis. Osteoporos Int 2006;17(8):1225-40.

9. Orsatti FL, Nahas EA, Maesta N, Nahas-Neto J, Burini RC. Plasma hormones, muscle mass and strength in resistance-trained postmenopausal women. Maturitas 2008; 20;59(4):394-404.

10. Mackey R, Eden J. Phytoestrogens and the menopause. Climacteric 1998;1(4):302-8.

11. Morito K, Hirose T, Kinjo J, Hirakawa T, Okawa M, Nohara T, et al. Interaction of phytoestrogens with estrogen receptors alpha and beta. Biol Pharm Bull 2001;24(4):351-6.

12. Setchell KD, Lydeking-Olsen E. Dietary phytoestrogens and their effect on bone: evidence from in vitro and in vivo, human observational, and dietary intervention studies. Am J Clin Nutr 2003;78(3 Suppl):593S-609S. 
13. Li B, Yu S. Genistein prevents bone resorption diseases by inhibiting bone resorption and stimulating bone formation. Biol Pharm Bull 2003;26(6):780-6.

14. Greendale GA, FitzGerald G, Huang MH, Sternfeld B, Gold E, Seeman T, et al. Dietary soy isoflavones and bone mineral density: results from the study of women's health across the nation. Am J Epidemiol 2002;155(8):746-54.

15. Lydeking-Olsen E, Beck-Jensen JE, Setchell KD, Holm-Jensen T. Soymilk or progesterone for prevention of bone loss-a 2 year randomized, placebo-controlled trial. Eur J Nutr 2004;43(4):246-57.

16. Ma DF, Qin LQ, Wang PY, Katoh R. Soy isoflavone intake increases bone mineral density in the spine of menopausal women: meta-analysis of randomized controlled trials. Clin Nutr 2008;27(1):57-64.

17. Gallagher JC, Satpathy R, Rafferty K, Haynatzka V. The effect of soy protein isolate on bone metabolism. Menopause 2004;11(3):290-8.

18. Brink E, Coxam V, Robins S, Wahala K, Cassidy A, Branca F. Long-term consumption of isoflavone-enriched foods does not affect bone mineral density, bone metabolism, or hormonal status in early postmenopausal women: a randomized, double-blind, placebo controlled study. Am J Clin Nutr 2008;87(3):761-70.

19. Nakagima D, Kim CS, Oh TW, Yang CY, Naka T, Igawa S, et al. Suppressive effects of genistein dosage and resistance exercise on bone loss in ovariectomizaded rats J Physiol Anthropol 2001;20(5):285-91.

20. Wu J, Wang X, Chiba H, Higuchi M, Nakatani T, Ezaki O, et al. Combined intervention of soy isoflavone and moderate exercise prevents body fat elevation and bone loss in ovariectomized mice. Metabolism 2004;53(7):942-8.

21. Wu J, Oka J, Tabata I, Higuchi M, Toda T, Fuku N, et al. Effects of isoflavone and exercise on BMD and fat mass in postmenopausal Japanese women: a 1-year randomized placebo-controlled trial. J Bone Miner Res 2006;21(5):780-9.

22. Wu J, Oka J, Higuchi M, Tabata I, Toda T, Fujioka M, et al. Cooperative effects of isoflavones and exercise on bone and lipid metabolism in postmenopausal Japanese women: a randomized placebo-controlled trial. Metabolism 2006;5:423-33.

23. Evans EM, Racette SB, Van Pelt RE, Peterson LR, Villareal DT. Effects of soy protein isolate and moderate exercise on bone turnover and bone mineral density in postmenopausal women. Menopause 2007;14(3 Pt 1):481-8.

24. Liu J, Ho SC, Su YX, Chen WQ, Zhang CX, Chen YM. Effect of long-term intervention of soy isoflavones on bone mineral density in women: a meta-analysis of randomized controlled trials. Bone 2009;44(5):948-53.

25. Marini H, Minutoli L, Polito F, Bitto A, Altavilla D, Atteritano M, et al. Effects of the phytoestrogen genistein on bone metabolism in osteopenic postmenopausal women: a randomized trial. Ann Intern Med 2007;146(12):839-47.

26. Cheong JM, Martin BR, Jackson GS, Elmore D, McCabe GP, Nolan JR, et al. Soy isoflavones do not affect bone resorption in postmenopausal women: a dose-response study using a novel approach with 41Ca. J Clin Endocrinol Metab 2007;92(2):577-82.

27. Wu J, Oka J, Ezaki J, Ohtomo T, Ueno T, Uchiyama S, et al. Possible role of equol status in the effects of isoflavone on bone and fat mass in postmenopausal Japanese women: a double-blind, randomized, controlled trial. Menopause 2007;14(5):866-74.

28. Robling AG, Castillo AB, Turner CH. Biomechanical and molecular regulation of bone remodeling. Annu Rev Biomed Eng 2006;8:455-98.

29. Chodzko-Zajko WJ, Proctor DN, Fiatarone Singh MA, Minson CT, Nigg CR, Salem GJ, et al. American College of Sports Medicine position stand. Exercise and physical activity for older adults. Med Sci Sports Exerc 2009;41(7):1510-30.

30. Asbury EA, Chandrruangphen P, Collins P. The importance of continued exercise participation in quality of life and psychological well-being in previously inactive postmenopausal women: a pilot study. Menopause 2006;13(4):561-7.
Endereço para correspondência

Fabio Lera Orsatti Universidade Federal do Triângulo Mineiro (UFTM)

Programa de Pós-Graduação em Educação Física/UFTM,

Avenida Tutunas, $n^{\circ} 490$ - Tutunas, CEP 38061-500, Uberaba, MG, Brasil. Fones: (34) 3318-5067 E-mail:fabiorsatti@gmail.com 